\title{
Process Monitoring Using Canonical correlation analysis
}

\author{
Shakir Muhammad SHAIKH ${ }^{3}$, Yin SHEN ${ }^{1}$, Shahid KARIM ${ }^{4}$, VISHAL KUMAR ${ }^{1}$ \\ 1 Department of Control Science and Engineering, Harbin Institute of Technology, Harbin, China \\ hit.shakir12@yahoo.com, shen.yin@hit.edu.cn,vishal@stu.hit.edu.cn, \\ 2 School of Computer Science and Technology, Harbin Institute of Technology \\ asiflaghari@hit.edu.cn \\ 4 School of Electronics and Information Engineering, Harbin Institute of Technology, Harbin, 150001, China shahidhit@yahoo.com
}

\begin{abstract}
Principal component analysis (PCA) and partial least square (PLS) used for fault diagnosis and process monitoring for systems. It is expected that the information to be examined isn't self-connected. However, the most largescale chemical industrial plants are nonlinear in nature so these techniques do not cope with them, invalid in nature. To fulfil the gap, there is need to develop an algorithm which can manage these nonlinearities of the process. The demands of industrial products are increasing rapidly so different adaptable techniques are being proposed. Canonical Correlation Analysis (CCA) is multivariate data-driven methodology which takes input-output both process data into consideration. Most industrial systems assumed that the data to be analysed is Gaussian in nature.However, it is not due to the nonlinearity'sreal systems in nature. In this work, an algorithm is developed that can monitor the system process using CCA with control limit that is achieved from the kernel density estimation by estimating probability density function (pdf).
\end{abstract}

Keywords:Canonical correlation Analysis, Process Monitoring, Kernel Density Estimation, Tennessee Eastman Process.

\section{Introduction}

In industrial systems data-driven techniques have extensively used for fault detection, and identification (FDI). In order to ensure safe and reliable operation and maintain product-quality, industrial process monitoring gained prominence in research and academia [1]. There owned technique multivariate analysis (MVA)is normally used to handle these kinds of problems [2].Principal component analysis (PCA) and partial least square (PLS) are Multivariable analysis techniques. It explores the correlation between interrelated variables that represent the system parameters. These techniques demonstrate several improvements against the univariate techniques [3]. Mostly industrial systems operate under changing operational conditions. Process monitoring aims to determine that fault has occurred or not. It is known as fault detection. A Fault is characterized as: 'any deviation of a boundary from ordinary condition' [4]. There are two steps in multivariate analysis technique (MVA): off-line training and online monitoring. PCA technique considers variables in both procedures and monitors deviation, occurs in usually process and actuator. PLS technique focuses on quality variables which are difficult to measure on-line or measure with a large delay. Process data is used to extract the latent variable in an off-line procedure, while in an online training process variables used to sense faults. When the opportunity arises to take into consideration both input and output data, CCA technique [5] is worthy to model fault diagnosis (FD) system. It is representative MVA technique but unfortunately seldom used for fault detection. CCA is extended form of PCA and PLS [6].Canonical variate analysis (CVA) is generalised form of CCA. Fault has been diagnosed with CVA which is published in [7]. It depends upon CVA variables reported in [8]. Fault detection residual signal is generated by CCA by considering input and output process data. Considering into account the connection between the incoming data and outgoing data variables, CCA technique come into being static and dynamic both process in steady state [9],[10]. A numerical example has been stated which generates random data to show the effectiveness of proposed algorithm. Later this algorithm tested on Tennessee Eastman process data. CCA algorithm with probability density estimation generates control limit which process variable cross and fault occurs. Normally control limit accepts that the cycle information follows the Gaussian information however in genuine the greater part of the interaction information are non-Gaussian in nature. CCA with KDE algorithm shows effectiveness and performance is evaluated. Mostly performance in these types of systems is evaluated by fault detection rate (FDR).

The remainder of paper is organized as follows. Area II exhibits the connected work.Section III demonstrates the implementation of CCA technique. Section IV is based on a numerical example. In section V TE process description and results are discussed. Finally section VI covers conclusion and future work. 


\section{Related work}

Over last eras, the prominent work on fault detection has received significant attention. Thus, automatic industrial process monitoring is an active area to study until now [11]. Extensively used data-driven based fault detection approaches, namely Multivariate Statistical Process Monitoring (MSPM) method which separates abnormal process from normal process conditions by forming statistical thresholds around feature variables take out from the process historical data [12]. Thus, early detection is valued for preemptive preservation of equipment and harmless operations. Two common categories of FD methods are model-based data-driven and others. Data-driven FD techniques have been extensively applied in industrial applications because these techniques take modest form and low demands on design effort. Instead, the industrial uses are commonly distinctive dynamic processes so might work on dissimilar functioning conditions in industrial situation. But, the multivariate statistical approaches could not be productively applied due to their assumption under stationary ideal operating conditions. The model-based systems need a priori mathematical knowledge of a process. Based on first principles process model is designed, the firm model-based techniques could be successfully applied. Then again, the information driven plans fill in as an effective way, wherein the fundamental cycle data can be taken out straightforwardly from the colossal measures of recorded interaction information. By considering the association between input-output variables, CCA methods have been established newly for static and dynamic both processes in steady state. The procedure whose center is the arrangement of the connection between two interaction factors, typically measure data sources and yields, may be seen as an expansion of the PLS and PCA techniques. These methods are dissimilar than CVA-based methods which rely on canonical variable [13]. CCA procedure for the most part settled by what strategy for standard connection based (RG) residual generation. The CCA techniques mainly considered by its expressively modest scheme because of avoiding the necessity for system identification which is required by CVA. Furthermore, the beneficial application of CCA method is based on the supposition that the process noises and subsequently the residual signal monitor a Gaussian distribution. In practice, fault detection considerably puzzles when the process under monitoring is non-Gaussian [14]. To deal with its challenges, few variants of the standing MVA techniques have been proposed. For the analysis of multimode nonlinear non Gaussian batch process, a novel model migration method has been proposed. In that method, PCA and ICA methods are combined [15]. An easy to implement method has been proposed in [16], which uses statistical method to diagnosis the fault and improve the system. Similar to this, another method is proposed in which fault relevant independent components extracted and fault commands in non-Gaussian and nonlinear processes [17]. Mutual information and ICA techniques together established for non-Gaussian process [18]. This method is to estimate the signal and then set threshold for fault detection by estimated distribution and this comes in the category of estimation-based. Gaussian mixture models (GMM) related methods [19], [20], Based on kernel methods [21], and consecutive quantile estimation [22] are illustrative examples. Although these methods have been applied effectively in complex system processes, their performance in FD are partial by choosing of appropriate kernel parameters and other quantified parameters. Hence, there is essential need to follow well-organized method based on the available measurements and threshold setting for non-Gaussian processes. Keeping in view above discussion, this paper focuses on CCA approach to detect faults in non-Gaussian processes. In this work control limit is achieved by kernel density estimation to cope with nonGaussian and this techniques is applied on CCA to generate residual signal, for fault diagnosis that proposed technique effectively have high fault detection rate (FDR) than of standard algorithm.

\section{Methodology}

\section{A. Canonical correlation analysis (CCA)}

CCA is a technique to capture the linear combinations of two vectors by maximizing correlations between them [23]. Suppose we have input-output data, that data is from process which is collected from the sensors of 
the system. Input is represented as $u$ and output asy. These input and output can be normalized as:

$\mathrm{u}=\left(\mathrm{u}_{\mathrm{obs}}-\mathrm{u}_{u}\right)$

$\mathrm{y}=\left(\mathrm{y}_{\mathrm{obs}}-\mathrm{u}_{\mathcal{y}}\right)$

$U=\left[u_{1}, u_{2}, \ldots, \mathrm{u}_{N}\right] \in \mathcal{R}^{\mathrm{l} \times \mathrm{N}}$

$Y=\left[y_{1}, y_{2}, \ldots, y_{N}\right] \in \mathcal{R}^{\mathrm{m} \times \mathrm{N}}$

$u_{i}$, and $y_{i}, i=1, \ldots, N$ are normalized to mean and their estimates are:

$\mathrm{u}_{u} \approx \frac{1}{\mathrm{~N}} \sum_{\mathrm{i}=1}^{\mathrm{N}} \mathrm{u}_{\mathrm{obs}}(\mathrm{i})$,

$\mathrm{u}_{\mathrm{y}} \approx \frac{1}{\mathrm{~N}} \sum_{\mathrm{i}=1}^{\mathrm{N}} \mathrm{y}_{\mathrm{obs}}(\mathrm{i})$,

Furthermore, the covariance $\left(\Sigma_{\mathrm{u}}\right),\left(\Sigma_{y}\right)$ and $\left(\Sigma_{u y}\right)$ calculated as:

$\Sigma_{u} \approx \frac{1}{N} \sum_{i=\mathrm{i}}^{N}\left(u_{o b s}(i)-u_{u}\right)$

$\Sigma_{\mathrm{u}} \approx(N-1)^{-1} \sum_{\mathrm{i}=1}^{\mathrm{N}}\left(\mathrm{u}_{\mathrm{obs}}(\mathrm{i})-\right.$

$\left.\mathrm{u}_{u}\right)\left(\mathrm{u}_{\mathrm{obs}}(\mathrm{i})-\mathrm{u}_{u}\right)^{\mathrm{T}}=\frac{\mathrm{UU}^{\mathrm{T}}}{\mathrm{N}-1}$

$\Sigma_{\mathrm{y}} \approx(N-1)^{-1} \sum_{\mathrm{i}=1}^{\mathrm{N}}\left(\mathrm{y}_{\mathrm{obs}}(\mathrm{i})-\right.$

$\left.\mathrm{u}_{\mathrm{y}}\right)\left(\mathrm{y}_{\mathrm{obs}}(\mathrm{i})-\mathrm{u}_{\mathrm{y}}\right)^{\mathrm{T}}=\frac{\mathrm{YY}^{\mathrm{T}}}{\mathrm{N}-1}$

$\Sigma_{\mathrm{uy}} \approx(N-1)^{-1} \sum_{\mathrm{i}=1}^{\mathrm{N}}\left(\mathrm{u}_{\mathrm{obs}}(\mathrm{i})-\right.$

$\left.\mathrm{u}_{u}\right)\left(\mathrm{u}_{\mathrm{obs}}(\mathrm{i})-\mathrm{u}_{\mathrm{y}}\right)^{\mathrm{T}}=\frac{\mathrm{UY}^{\mathrm{T}}}{\mathrm{N}-1}$

with CCA technique, it turns out that:

$$
\begin{aligned}
\left(\frac{U U^{T}}{N-1}\right)^{-\frac{1}{2}}\left(\frac{U Y^{T}}{N-1}\right) & \left(\frac{Y Y^{T}}{N-1}\right)^{-\frac{1}{2}} \\
= & \Sigma_{u}{ }^{-\frac{1}{2}} \Sigma_{u y} \Sigma_{y}{ }^{-\frac{1}{2}} \\
= & \Gamma_{s} \Sigma Y_{s}^{T},
\end{aligned}
$$

Where $\Sigma=\left[\begin{array}{cc}\Sigma_{\varrho} & 0 \\ 0 & 0\end{array}\right] \operatorname{rank} \Sigma_{u y}=\varrho$, and $\Sigma_{\varrho}=$ $\operatorname{diag}\left(p_{1}, \ldots, p_{\varrho}\right) \Gamma=\left(w_{1}, \ldots w_{d_{1}}\right) \Upsilon=$ $\left(V_{1}, \ldots, V_{d_{2}}\right)$

Let:

$\mathrm{J}_{\mathrm{s}}=\Sigma_{\mathrm{u}}{ }^{-\frac{1}{2}} \Gamma(:, 1: \varrho)$,

$\mathrm{L}_{\mathrm{s}}=\Sigma_{\mathrm{y}}{ }^{-\frac{1}{2}} \Upsilon(:, 1: \mathrm{Q})$

$\mathrm{J}_{\text {res }}=\Sigma_{\mathrm{u}}{ }^{-\frac{1}{2}} \Gamma(:, \varrho+1: \mathrm{l})$,

$\mathrm{L}_{\mathrm{res}}=\Sigma_{y}{ }^{-\frac{1}{2}} \Upsilon(:, \varrho+1: m)$

Relationship between $L_{S}^{T}$ and $J_{S}^{T}$ break down into:

$\mathrm{L}_{\mathrm{s}}^{\mathrm{T}} \mathrm{y}(\mathrm{k})=\Sigma_{\mathrm{Q}} J_{s}^{\mathrm{T}} \mathrm{u}(\mathrm{k})+\mathrm{V}_{\mathrm{s}}(\mathrm{k})$

$V_{s}$ is measurement noise, dimly correlated with $J_{s}^{T} u$. As a result, residual vector define as:

$\mathrm{r}_{(\mathrm{k})}=\mathrm{L}_{\mathrm{s}}^{\mathrm{T}} \mathrm{y}(\mathrm{k})-\mathrm{M}_{\mathrm{s}}^{\mathrm{T}} \mathrm{u}(\mathrm{k})$

$\mathrm{M}_{\mathrm{s}}^{\mathrm{T}}=\Sigma_{\mathrm{\varrho}} \mathrm{J}_{\mathrm{s}}^{\mathrm{T}}$

Where $r$ will follow a normal distribution. $r(k)$ ' $\mathrm{s}$ covariance is estimated by:

http://www.sic.ici.ro
$(N-1)^{-1}\left(L_{s}^{T} Y-\Sigma_{\varrho} J_{s}^{T} U\right)\left(L_{S}^{T} Y-\right.$

$\left.\Sigma_{\varrho} J_{S}^{T} U\right)^{T}=\frac{I_{e}-\Sigma_{\varrho}^{2}}{N-1}$

Test Statistics

$\mathrm{T}_{\mathrm{cca}}^{2}(\mathrm{k})=(\mathrm{N}-1) \mathrm{r}^{\mathrm{T}}(\mathrm{k})\left(\mathrm{I}_{\mathrm{e}}-\Sigma_{\mathrm{Q}}^{2}\right)^{-1} \mathrm{r}(\mathrm{k})$

The Threshold value $\mathrm{T}^{2}$ for a given level of significance $\alpha$ likeP $\left(\mathrm{T}^{2}<\mathrm{T}^{2}{ }_{\text {th }}(\alpha)\right)=$ $\alpha$.Normally these threshold values follow along with Gaussian distribution. However, this assumption is invalid due to the most industrial process are nonlinear. An eminent strategy built up that adapt to this matter of conflict by first assuming the (pdf) probability density function of the factual estimations with the assistance of (kdf) kernel density estimations.. The

likelihood $\mathrm{x}$ with $\mathrm{p}(\mathrm{x}) \mathrm{i}, \mathrm{e}$ arbitrary variable with (pdf) probability density function to be lesser than a guaranteed esteem $\mathrm{s}$ is decide as:

\section{B. Calculation of CCA based process monitoring control limit}

Kernel density estimation is proposed in this work in order to cope with Gaussian assumption. Mostly control limit is calculated by Gaussian assumption in this work. We propose an algorithm that uses probability density function using kernel density estimation to evaluate nonlinearities in the process.

$p(x<s)=\int_{-\infty}^{s} p(x) d x$

Where $p(x)$ can be predicated from $\mathrm{K}$, kernel function:

$$
\begin{aligned}
& p(x)=\frac{1}{M h} \sum_{k=1}^{M} K\left(\frac{x-x_{k}}{h}\right) \\
& k(g)=\frac{e^{\frac{-g^{2}}{2}}}{\sqrt{2 \pi}}
\end{aligned}
$$

In the equation, $h$ is the calculated bandwidth and $x_{k}$ is sample of $x$. By substituting $x_{k}$ with $T_{k}^{2}$ obtained from (19). The corresponding threshold for these data correspond to $s$ in (20), acquired by solving $P(x<s)=\alpha$. The $T^{2}$ cadent means necessary deviation of sanctioned factors exhibit change in the expansion of head subspace. $T^{2}$ Metric is important; faults can source an inconsistent growth in the principal space and other will be revealed as; an increase in the residual variability space. A Fault could happen if the statistics exceed the threshold value. Following the way, the quantities of the measurements determined at the hour of preparing stages which are agent for the ordinary framework when no shortcoming had occur. The edge is assessed for an ostensible 
move of effect by seeing at the likelihood thickness capacity of these insights, arriving at the edge and vulnerability occurs. Any deviancy in the lead of framework elements delivered by pass, will influence an expansion in the measure of these insights traversing the edge and making an alarm [23].In an internet preparing information is prototypal, the calculation be sufficient to oblige estimation of measurements beneath the edge in the event that if no issues are accessible. And in testing phase of the algorithm the new data is generated which is not included in training model could be or not faulty. However two procedures are there for testing data, one is online and other is off-line. In this work we will focus on off-line training and testing data, later one is adaptive approach.

\section{A Numerical Example}

In this section numerical supposition has been studied. The effectiveness of CCA algorithm with KDE has been demonstrated on randomly generated data. These methods provide that algorithms working well and later it will be shown to industrial benchmark. To train model, two thousand samples are generated. It is establish that $L^{T} y$ and $J^{T} u$ have strong correlation the confidence limit is 0.01 . A fault has occurred after 1000 samples, which is apparently shown in figure 1. An input output linear relationship is given in a numerical example which is modeled as:

$y(k)=\Psi \mathrm{u}(\mathrm{k})+\mathrm{b}_{o b s}+v(k)$

Where $u \in \mathcal{R}^{l}, y \in \mathcal{R}^{m}$ are input and output variables $\Psi$ is system parameter

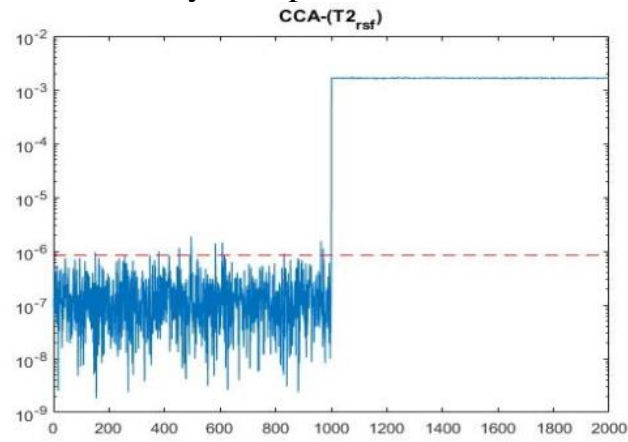

Figure 1: Random fault scenario

\section{Description of Tennessee Eastman process}

The TEP is designed to simulate that create real time chemical producing process. It has been used a lot in many academic and research work as a benchmark to show various algorithms. $[25,26]$. A test system is created, subtleties is in [26]. In this piece of paper, CCA with KDE which is proposed calculation for issue conclusion exhibit on Tennessee Eastman measure (TEP) to. It is a down to earth modern cycle. TE measure filled in as received benchmark. The fundamental model of TE measure is apply to assess the essential Multivariate Statistical Process Monitoring strategies under ideal fixed working conditions. Figure 2 shows stream of interaction with 5 significant sections. Cycle has four reactants and 2 items. Alongside a latent and a result are likewise accessible, detailing an aggregate of 8 segments. The interaction own altogether 52 measurements out of which 11 are controlled factors and 41 are measure factors. In noticed deficiencies, which can turn out all the while.A. Application to TEP

The proposed algorithm of CCA with KDE is applied to Tennessee Eastman process (TEP) in this section. It contains the data that is received from sensors, 52 variables and also 960 measurements. In this case study fault no 1, 3 and 5 are under consideration.

\section{B. Test setup and Training}

Inputs are in this work, the manipulated variables, and for outputs (XMEAS1XMEAS31) are selected as given in table 2. In the training phase these variables are chosen for input-output patterns which are used for training with CCA.

\section{Test results}

In test results, it is shown that the fault detection rate is higher for CCA with KDE than forstandard CCA. As a representative fault no 1,3 and 5 simulated and results are shown in figure 3, 4 and 5 respectively. In this dataset, 960 samples of 52 variables, fault occurred at 160 sample of data set.

$$
F D R=\frac{\text { Number of samples }\left(j>j_{\text {th }} \mid \text { fault free }\right)}{\text { total number of sampling }}
$$




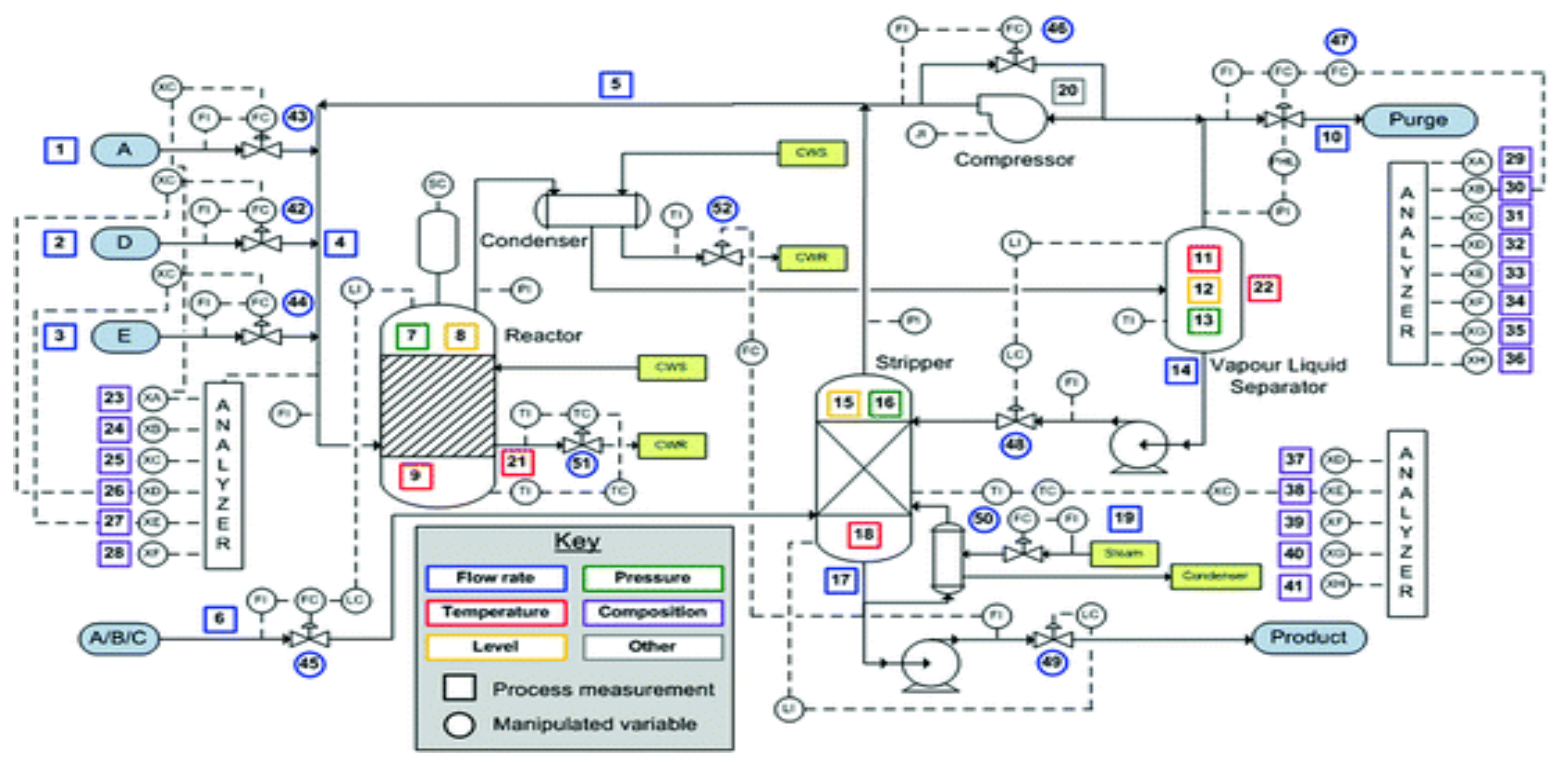

Figure 2. Tennessee Eastman Process [27]

Table 1. List of process and manipulated Variables

\begin{tabular}{|l|l|}
\hline Tag & Description \\
\hline XMV(1) & D feed flow \\
XMV(2) & E feed flow \\
\hline XMV(3) & A feed flow \\
\hline XMV(4) & A and C feed flow \\
\hline XMV(6) & Purge Valve \\
XMV(7) & Separator pot liquidflow \\
\hline XMV(8) & Stripper liquid product flow \\
\hline XMV(10) & Reactor Cooling water flow \\
\hline XMV(11) & Condenser Cooling water flow \\
XMEASV(1) & A feed (Stream 1) \\
\hline XMEASV(2) & D feed (Stream 2) \\
\hline XMEASV(3) & E feed (Stream 3) \\
\hline XMEASV(4) & A and C feed \\
XMEASV(5) & Recycle flow \\
\hline XMEASV(6) & Reactor feed rate \\
\hline XMEASV(7) & Reactor Pressure \\
\hline XMEASV(8) & Reactor level \\
\hline XMEASV(9) & Reactor temperature \\
XMEASV(10) & Purge rate \\
\hline XMEASV(11) & Separator temperature \\
\hline XMEASV(12) & Separator level \\
\hline XMEASV(13) & Separator pressure \\
XMEASV(14) & Separator underflow \\
\hline XMEASV(15) & Stripper level \\
\hline XMEASV(16) & Stripper pressure \\
\hline XMEASV(17) & Stripper underflow \\
XMEASV(18) & Stripper temperature \\
\hline XMEASV(19) & Stripper steam flow \\
\hline XMEASV(20) & Compressor work \\
\hline XMEASV(21) & Reactor water temperature \\
XMEASV(22) & Separator water temperature \\
\hline
\end{tabular}

Table 2. List of Process Manipulated Variables in Tennesseans Eastman Process

\begin{tabular}{|l|l|l|l|}
\hline $\begin{array}{l}\text { Variable } \\
\text { name }\end{array}$ & Number & $\begin{array}{l}\text { Base } \\
\text { Value } \\
(\%)\end{array}$ & Units \\
\hline D feed flow & $\mathrm{XMV}(1)$ & 63.053 & $\mathrm{kgh}^{-1}$ \\
\hline E feed flow & $\mathrm{XMV}(2)$ & 53.980 & $\mathrm{kgh}^{-1}$ \\
\hline A feed flow & $\mathrm{XMV}(3)$ & 24.644 & $\mathrm{kscmh}$ \\
\hline $\begin{array}{l}\text { A and C feed } \\
\text { flow }\end{array}$ & $\mathrm{XMV}(4)$ & 61.302 & $\mathrm{kscmh}$ \\
\hline $\begin{array}{l}\text { Compressor } \\
\text { recycle valve }\end{array}$ & $\mathrm{XMV}(5)$ & 22.210 & $\%$ \\
\hline $\begin{array}{l}\text { Purge valve } \\
\text { Separatorpot } \\
\text { liquid flow }\end{array}$ & $\mathrm{XMV}(7)$ & 38.100 & $\%$ \\
\hline $\begin{array}{l}\text { Stripper liquid } \\
\text { product flow }\end{array}$ & $\mathrm{XMV}(8)$ & 46.534 & $\mathrm{~m}^{3} \mathrm{~h}^{-1}$ \\
\hline $\begin{array}{l}\text { Stripper steam } \\
\text { valve }\end{array}$ & $\mathrm{XMV}(9)$ & 47.446 & $\%$ \\
\hline $\begin{array}{l}\text { Reactor } \\
\text { cooling water } \\
\text { flow }\end{array}$ & $\mathrm{XMV}(10)$ & 41.106 & $\mathrm{~m}^{3} \mathrm{~h}^{-1}$ \\
\hline Condenser & $\mathrm{XMV}(11)$ & 18.114 & $\mathrm{~m}^{3} \mathrm{~h}^{-1}$ \\
\hline
\end{tabular}

\section{Algorithm for CCA with KDE fault detection}

\section{Off-line design}

Step1: Collect input-output data uandy

Step 2: centre the process input output data (16)

Step3: Doing SVD on input output data using (11)

\section{On-line implementation}

Step 1: Collect the input- output (online)

Step 2: Calculate the residual statistics using eq (17)

Step 3: Calculate the $\boldsymbol{T}^{2}$ statistics using eq (20)

Step 4: Based on decision logic take decision: 
Step 5: $T_{k d e}^{2}>j_{t h} \Rightarrow$ faulty, otherwisefaultfree

Table 3. List of Faults in Tennessee Eastman Process

\begin{tabular}{|c|c|c|}
\hline $\begin{array}{l}\text { Fault } \\
\text { number }\end{array}$ & Process Variable & Type \\
\hline IDV(1) & $\begin{array}{l}\text { A/C feed ratio, B } \\
\text { composition constant }\end{array}$ & Step \\
\hline IDV(2) & $\begin{array}{l}\text { B composition, } A / C \\
\text { ration constant }\end{array}$ & Step \\
\hline IDV(3) & D feed temperature & Step \\
\hline IDV(4) & $\begin{array}{l}\text { Reactor cooling } \\
\text { water inlet } \\
\text { temperature }\end{array}$ & Step \\
\hline IDV(5) & $\begin{array}{l}\text { Condenser cooling } \\
\text { water inlet } \\
\text { temperature }\end{array}$ & Step \\
\hline IDV(6) & A feedloss & Step \\
\hline IDV(7) & $\begin{array}{l}\text { Cheader pres sure } \\
\text { loss-reduced } \\
\text { availability }\end{array}$ & Step \\
\hline IDV(8) & $\begin{array}{l}\text { A, BC feed } \\
\text { composition }\end{array}$ & $\begin{array}{l}\text { Random } \\
\text { variation }\end{array}$ \\
\hline IDV(9) & D feed temperature & $\begin{array}{l}\text { Random } \\
\text { variation }\end{array}$ \\
\hline IDV(10) & C feed temperature & $\begin{array}{l}\text { Random } \\
\text { variation }\end{array}$ \\
\hline IDV(11) & $\begin{array}{l}\text { Reactor cooling } \\
\text { water inlet } \\
\text { temperature }\end{array}$ & $\begin{array}{l}\text { Random } \\
\text { variation }\end{array}$ \\
\hline IDV(12) & $\begin{array}{l}\text { Condenser cooling } \\
\text { water valve }\end{array}$ & $\begin{array}{l}\text { Random } \\
\text { variation }\end{array}$ \\
\hline IDV(13) & Reaction Kinetics & Slow Drift \\
\hline IDV(14) & $\begin{array}{l}\text { Reactor cooling } \\
\text { water valve }\end{array}$ & Sticking \\
\hline IDV(15) & $\begin{array}{l}\text { Condenser cooling } \\
\text { water valve }\end{array}$ & Sticking \\
\hline IDV(16) & Unknown & Unknown \\
\hline IDV(17) & Unknown & Unknown \\
\hline IDV(18) & Unknown & Unknown \\
\hline IDV(19) & Unknown & Unknown \\
\hline IDV (20) & Unknown & Unknown \\
\hline IDV(2I) & $\begin{array}{l}\text { The valve fixedat } \\
\text { steady state position }\end{array}$ & $\begin{array}{l}\text { Constant } \\
\text { Position }\end{array}$ \\
\hline
\end{tabular}

Table 4: Detection results in terms of FDR

\begin{tabular}{|l|l|l|}
\hline Fault IDs: & CCA with KDE & CCA \\
\hline IDV(1) & $99.12 \%$ & $88.62 \%$ \\
\hline IDV(2) & $99.13 \%$ & $93.24 \%$ \\
\hline IDV(5) & $99.75 \%$ & $99.62 \%$ \\
\hline
\end{tabular}

It is shown in above results that the CCA with KDE have higher FDR than CCA standard algorithm.

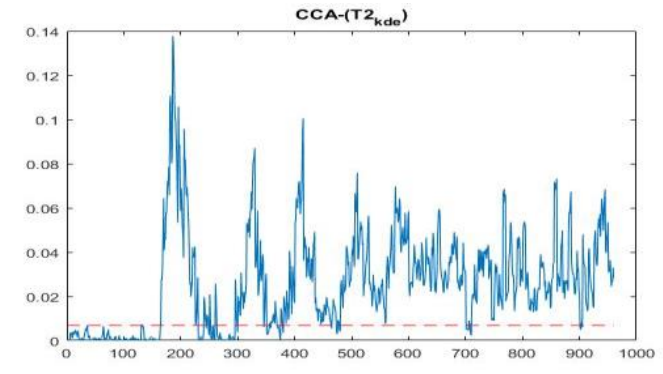

Figure 3. Fault scenario 1

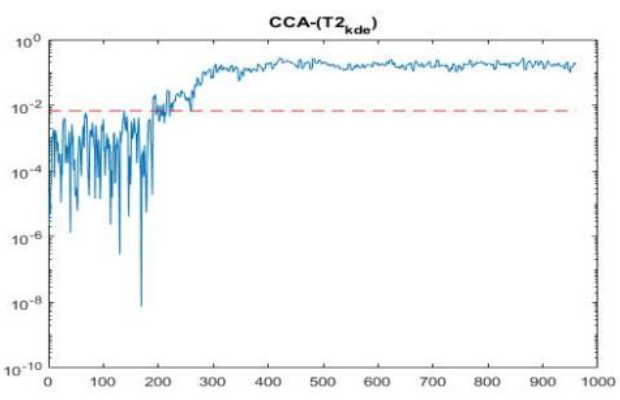

Figure 4. Fault scenario 2

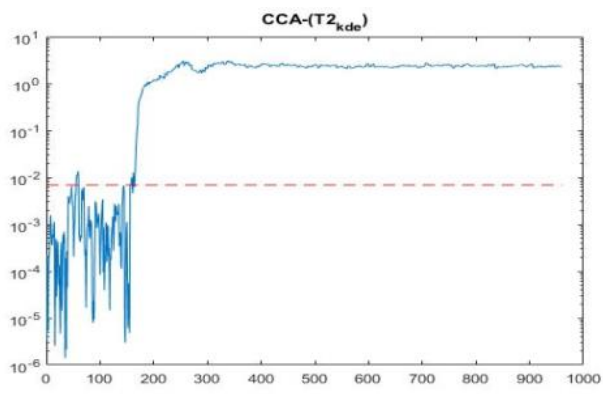

Figure 5. Fault scenario 5

\section{Conclusion and future work}

In this paper, improved CCA based issue analysis methods have been examined. At that point, standard CCA strategy applied on arbitrary information to show the adequacy of the calculation. The prevailing methods for the first time have been dealt with Gaussian and non-Gaussian assumption of industrial process data. Despite the achievements of data-driven algorithms in the application of fault detection and diagnosis using industrial simulated system based on the assumption of Gaussian, there is lack of non-Gaussian work. This work is presented with aim to cope with real simulated data from industries which are usually non Gaussian in nature and effectiveness of these algorithms applied on Tennessee Eastman industrial simulated plant. In this case study three faults have been simulated. The faults are effectively detected using the $T^{2}$ statistics. These outcomes approve that CCA can be proficiently applied for shortcoming location and analysis in genuine complex frameworks working under adaptable working conditions. The CCA system when contrast a presentation and CCA-KDE it is perfect that the strategy is better than the others as far as faut detectin rate (FDR). It is worthy to note that the procedure of $\mathrm{KDE}$ is essential for threshold estimation otherwise Gaussian assumption was impractical due to nonlinearities in the process plant system. Despite of quite satisfactory 
performance demonstrated by CCA along with $\mathrm{KDE}$, the fault detection in highly dynamic nonlinear is fairly challenging, which force todevelop improved algorithms for these particular faults in future.

\section{REFERENCES}

1.Isermann, Rolf. "Fault detection with Principal Component Analysis (PCA)." FaultDiagnosis Systems. Springer, Berlin, Heidelberg, 2006. 267-278.

2. Ge, Zhiqiang. "Review on data-driven modeling and monitoring for plant-wide industrial processes." Chemometrics and Intelligent Laboratory Systems (2017).

3. Odiowei, P. P., and Y. Cao. "Nonlinear dynamic process monitoring using canonical variate analysis and kernel density estimations." Computer Aided Chemical Engineering. Vol. 27. Elsevier, 2009. 15571562.

4. Isermann, Rolf. "Model-based faultdetection and diagnosis-status and applications." Annual Reviews in control 29.1 (2005): 71-85.

5. Hotelling, Harold. "Relations between two sets of variates." Biometrika 28.3/4 (1936): 321-377.

6. Li, Gang, S. Joe Qin, and Donghua Zhou. "Geometric properties of partial least squares for process monitoring." Automatica 46.1 (2010): 204-210

7.Chiang, Leo H., Evan L. Russell, and Richard D. Braatz. Fault detection and diagnosis in industrial systems. Springer Science \& Business Media, 2000.

8. Yin, Shen, Lei Liu, and Jian Hou. "A multivariate statistical combination forecasting method for product quality evaluation." Information Sciences 355 (2016): 229-236.

9. Chen, Zhiwen, et al. "Canonical correlation analysis-based fault detection methods with application to alumina evaporation process." Control Engineering Practice 46 (2016): 51-58.

10. Chen, Zhiwen. Data-driven Fault Detection for Industrial Processes: Canonical Correlation Analysis and Projection Based Methods. Springer, 2017.

11. Reis, Marco S., and Geert Gins. "Industrial Process Monitoring in the Big Data/Industry
4.0 Era: From Detection, to Diagnosis, to Prognosis." Processes 5.3 (2017): 35.

12. Chiang, Leo H., Evan L. Russell, and Richard D. Braatz. Fault detection and diagnosis in industrial systems. Springer Science \& Business Media, 2000.

13. Larimore, Wallace E. "System identification, reduced-order filtering and modeling via canonical variate analysis." American Control Conference, 1983. IEEE, 1983.

14. Yin, Shen, et al. "A review on basic datadriven approaches for industrial process monitoring." IEEE Transactions on Industrial Electronics 61.11 (2014): 6418-6428.

15. Zhang, Yingwei, Jiayu An, and Chi Ma. "Fault detection of non-Gaussian processes based on model migration." IEEE Transactions on Control Systems Technology 21.5 (2013): 1517-1526.

Zhang, Yingwei, Nan Yang, and Shipeng Li.

16.Chenaru, Oana, et al. "Fault-Tolerant Control System Implementation Based on Parameter Analysis." analysis 4.6 (2016): 18.

17."Fault isolation of nonlinear processes based on fault directions and features." IEEE Transactions on Control Systems Technology 22.4 (2014): 1567-1572.

18. Rashid, Mudassir M., and Jie Yu. "A new dissimilarity method integrating multidimensional mutual information and independent component analysis for nonGaussian dynamic process monitoring." Chemometrics and Intelligent Laboratory Systems 115 (2012): 44-58.

19.C. M. Bishop, Pattern Recognition and Machine Learning (Information Science and Statistics). Secaucus, NJ, USA: SpringerVerlag, 2006

20. Chen, Tao, Julian Morris, and Elaine Martin. "Probability density estimation via an infinite Gaussian mixture model: application to statistical process monitoring." Journal of the Royal Statistical Society: Series C (Applied Statistics) 55.5 (2006): 699-715.

21. Odiowei, Pabara-Ebiere Patricia, and Yi Cao. "Nonlinear dynamic process monitoring using canonical variate analysis and kernel density estimations." IEEE Transactions on Industrial Informatics 6.1 (2010): 36-45.

22. Tschumitschew, Katharina, and Frank Klawonn. "Incremental quantile estimation." Evolving Systems 1.4 (2010): 253264. 
23. Hardoon, David R., Sandor Szedmak, and John Shawe-Taylor. "Canonical correlation analysis: An overview with application to learning methods." Neural computation 16.12 (2004): 2639-2664.

24. Chen, Zhiwen, et al. "Fault Detection for Non-Gaussian Processes Using Generalized Canonical Correlation Analysis and Randomized Algorithms." IEEE Transactions on Industrial Electronics 65.2 (2018): 15591567.

25.Yin, Shen, et al. "A comparison study of basic data-driven fault diagnosis and process monitoring methods on the benchmark Tennessee Eastman process." Journal of Process Control22.9 (2012): 1567-1581.

26. Ricker, N. Lawrence. "Decentralized control of the Tennessee Eastman challenge process." Journal of Process Control6.4 (1996): 205-221.

27. Chen, Zhiwen, et al. "Improved canonical correlation analysis-based fault detection methods for industrial processes." Journal of Process Control 41 (2016): 26-34. 